\title{
\begin{tabular}{l|l} 
Mibraries & DSpace@MIT
\end{tabular}
}

\author{
MIT Open Access Articles
}

\section{Adiabatic Green's function technique and transient behavior in time-dependent fermion-boson coupled models}

The MIT Faculty has made this article openly available. Please share how this access benefits you. Your story matters.

Citation: Oh, Yun-Tak; Higashi, Yoichi; Chan, Ching-Kit and Han, Jung Hoon. "Adiabatic Green's function technique and transient behavior in time-dependent fermion-boson coupled models." Physical Review B 94, 075113 (August 2016): 1-10 (C) 2016 American Physical Society

As Published: http://dx.doi.org/10.1103/PhysRevB.94.075113

Publisher: American Physical Society

Persistent URL: http://hdl.handle.net/1721.1/110390

Version: Final published version: final published article, as it appeared in a journal, conference proceedings, or other formally published context

Terms of Use: Article is made available in accordance with the publisher's policy and may be subject to US copyright law. Please refer to the publisher's site for terms of use. 


\title{
Adiabatic Green's function technique and transient behavior in time-dependent fermion-boson coupled models
}

\author{
Yun-Tak Oh, ${ }^{1}$ Yoichi Higashi, ${ }^{1}$ Ching-Kit Chan, ${ }^{2}$ and Jung Hoon Han ${ }^{1, *}$ \\ ${ }^{1}$ Department of Physics, Sungkyunkwan University, Suwon 16419, Korea \\ ${ }^{2}$ Department of Physics, Massachusetts Institute of Technology, Cambridge, Massachusetts 02139, USA
}

(Received 20 January 2016; revised manuscript received 12 July 2016; published 8 August 2016)

\begin{abstract}
The Lang-Firsov Hamiltonian, a well-known solvable model of interacting fermion-boson system with sideband features in the fermion spectral weight, is generalized to have the time-dependent fermion-boson coupling constant. We show how to derive the two-time Green's function for the time-dependent problem in the adiabatic limit, defined as the slow temporal variation of the coupling over the characteristic oscillator period. The idea we use in deriving the Green's function is akin to the use of instantaneous basis states in solving the adiabatic evolution problem in quantum mechanics. With such "adiabatic Green's function" at hand we analyze the transient behavior of the spectral weight as the coupling is gradually tuned to zero. Time-dependent generalization of a related model, the spin-boson Hamiltonian, is analyzed in the same way. In both cases the sidebands arising from the fermion-boson coupling can be seen to gradually lose their spectral weights over time. Connections of our solution to the two-dimensional Dirac electrons coupled to quantized photons are discussed.
\end{abstract}

DOI: 10.1103/PhysRevB.94.075113

\section{INTRODUCTION}

Time-dependent quantum-mechanical phenomena have interested scientists since the inception of quantum mechanics. A rare example of an exactly solvable time-dependent problem was discovered as early as 1932, known as the LandauMajorana-Zener problem [1-3]. A particular class of timedependent problems in which the Hamiltonian is periodic in time, $H(t+T)=H(t)$, can be treated in the Floquet framework [4,5]. Generalizations of the Floquet theory that include the coupling to the dissipative reservoir and the quench of the periodic drive have been studied extensively in the past [6-19].

The other limit in which the time-dependent problem becomes tractable is when the temporal variation is slow, or "adiabatic." A general strategy for treating the adiabatic evolution of the quantum system was laid out by Berry [20]. The basic idea there was to expand the quasiexact eigenstate in the instantaneous basis $\left|\phi_{n}(t)\right\rangle$, defined by the eigenvalue problem

$$
H(t)\left|\phi_{n}(t)\right\rangle=E_{n}(t)\left|\phi_{n}(t)\right\rangle
$$

for each time slice $t$. It is implicit in carrying out Berry's program that one has the solutions of the instantaneous Hamiltonian $H(t)$ at hand. Berry's idea is most often applied to the single-particle evolution under a parametrically slow external drive, but the idea itself is general enough to apply to an arbitrary many-body problem, provided a well-defined gap separates the ground state from the first excited state at all times. For many-body problems it is often more useful to work with the Green's function containing information for all energies, instead of the wave function that addresses the ground state property only. We show how to derive the nonequilibrium Green's function in the adiabatic limit, for a simple time-dependent many-body model. Explicitly, we work

\footnotetext{
*hanjh@skku.edu
}

with the time-dependent generalization of the exactly solvable Lang-Firsov (LF) model [21] and a related, spin-boson (SB) model [22-24]. Due to the time dependence of the Hamiltonian the two-time Green's function becomes dependent on the two times separately. Most often, calculation of the nonequilibrium two-time Green's function is done by the Keldysh technique $[25,26]$. We show, in the adiabatic limit of the time-dependent Lang-Firsov model, how to obtain the two-time Green's function without the reliance on the Keldysh method.

Stripped down to its bare minimum, the LF model contains a single fermionic level coupled to a single harmonic oscillator of frequency $\omega_{0}$. The exact single particle Green's function, obtained through a canonical transformation method, shows in its imaginary part a series of delta function peaks spaced at intervals of $\hbar \omega_{0}$ [27]. Each $n$th delta function represents a fermionic level dressed by $n$ bosons. When the fermion-boson coupling is turned off, the series of delta functions will reduce to a single peak at the fermion energy. How the evolution from multiple peaks to a single peak takes place, as the coupling is gradually turned to zero, is the question we are going to address quantitatively with the adiabatic Green's function method.

In Sec. II, we begin by introducing a time-dependent variant of the LF Hamiltonian and outline how to derive the Green's function for it. Complex details of the derivation can be found in Appendix A. Recent developments in pump-probe techniques have made it possible to observe real-time dynamics of the band electrons under the influence of the intense pump laser. The time-resolved photoemission spectroscopy can be calculated with the lesser Green's function for the system [28]. We calculate the photocurrent for the time-dependent LF model based on our calculation of the lesser Green's function in Sec. III, with emphasis on how the sidebands decay over time as the fermion-boson coupling is gradually turned off. In Sec. III B, we solve the time-dependent version of the spin-boson model. It is our hope that the methodology developed in this paper can be further generalized to solve the problem of sideband decay in real materials such as the surface of topological insulators [29,30]. A brief discussion 
of the potential relevance of our work to the two-dimensional Dirac electrons coupled to quantized photon fields is given in Sec. III C. A summary and outlook follows in Sec. IV.

\section{TIME-DEPENDENT LANG-FIRSOV HAMILTONIAN}

\section{A. Model}

The Lang-Firsov Hamiltonian

$$
H=\varepsilon c^{\dagger} c+\omega_{0} a^{\dagger} a+g \omega_{0} c^{\dagger} c\left(a+a^{\dagger}\right)
$$

expresses the coupling of a fermionic level of energy $\varepsilon$ interacting with the harmonic oscillator mode of frequency $\omega_{0}$. It is diagonalized by the unitary operator $\mathcal{U}$ :

$$
\begin{aligned}
& \mathcal{U}=e^{g c^{\dagger} c\left(a-a^{\dagger}\right)}, \\
& \bar{H}=\mathcal{U}^{\dagger} H \mathcal{U}=\bar{\varepsilon} c^{\dagger} c+\omega_{0} a^{\dagger} a,
\end{aligned}
$$

with the renormalized energy $\bar{\varepsilon}=\varepsilon-g^{2} \omega_{0}$. The unitary operator $\mathcal{U}$ transforms the boson and fermion operators

$$
\mathcal{U}^{\dagger} a \mathcal{U}=a-g c^{\dagger} c, \quad \mathcal{U}^{\dagger} c \mathcal{U}=c X,
$$

where one can recognize $X=e^{g\left(a-a^{\dagger}\right)}$ as the coherent state operator,

$$
X^{\dagger} a X=a-g, \quad X^{\dagger}|\alpha\rangle=e^{-\frac{g}{2}\left(\alpha-\alpha^{*}\right)}|\alpha+g\rangle .
$$

The factor $e^{-(g / 2)\left(\alpha-\alpha^{*}\right)}$ is a pure phase and we have introduced the coherent state $|\alpha\rangle: a|\alpha\rangle=\alpha|\alpha\rangle$.

The fermion Green's function for the Lang-Firsov model can be obtained exactly thanks to the existence of a unitary operator $\mathcal{U}$. For instance, the greater Green's function

$$
G^{>}\left(t, t^{\prime}\right)=-i \operatorname{Tr}\left[c(t) c^{\dagger}\left(t^{\prime}\right) \rho\right],
$$

where $\rho$ is the density matrix giving the initial preparation of the fermion-boson state at time $t_{0}$, and $c(t)=$ $e^{i H\left(t-t_{0}\right)} c e^{-i H\left(t-t_{0}\right)}$ is the Heisenberg operator, can be obtained exactly for the initial density matrix

$$
\rho=|\alpha\rangle\langle\alpha| .
$$

We set empty fermion state in the initial density matrix because the occupied fermion state gives zero to Eq. (2.5). A straightforward calculation finds

$$
G^{>}\left(t, t^{\prime}\right)=-i e^{-i \bar{\varepsilon}\left(t-t^{\prime}\right)} e^{g^{2}\left(e^{-i \omega_{0}\left(t-t^{\prime}\right)}-1\right)} e^{\left(\alpha-\alpha^{*}\right)\left(g(t)-g\left(t^{\prime}\right)\right)},
$$

where $g(t)=g e^{i \omega_{0}\left(t-t_{0}\right)}$. When $\alpha=0$, it reduces to the wellknown form

$$
G^{>}\left(t-t^{\prime}\right)=e^{-i \bar{\varepsilon}\left(t-t^{\prime}\right)-g^{2}} \sum_{n=0}^{\infty} g^{2 n} \frac{e^{-i n \omega_{0}\left(t-t^{\prime}\right)}}{n !},
$$

that gives a series of delta-function peaks of weights $g^{2 n} / n$ ! for the $n$th sideband.

We now generalize the Lang-Firsov model to include the explicit time dependence in the coupling constant, $g \rightarrow g(t)$ :

$$
H(t)=\varepsilon c^{\dagger} c+\omega_{0} a^{\dagger} a+g(t) \omega_{0} c^{\dagger} c\left(a+a^{\dagger}\right) .
$$

This $g(t)$ is not the same factor $g(t)$ appearing in Eq. (2.7). Rather, it is a genuine time-dependent fermion-boson coupling $g(t)$ that, by assumption, varies slowly on the time scale of the oscillator $\tau_{0}=2 \pi / \omega_{0}$,

$$
\left|g^{\prime}(t)\right| \tau_{0} \ll|g(t)|,
$$

where $g^{\prime}(t)$ is the temporal derivative of $g(t)$. The Green's function (2.5) for the time-dependent LF model is

$$
G^{>}\left(t, t^{\prime}\right)=-i\left\langle\alpha\left|U\left(t_{0}, t\right) c U\left(t, t^{\prime}\right) c^{\dagger} U\left(t^{\prime}, t_{0}\right)\right| \alpha\right\rangle .
$$

The initial time $t_{0}$ is usually set to the distant past $t_{0} \rightarrow-\infty$. The evolution operator $U\left(t, t^{\prime}\right)$, not to be confused with the unitary operator $\mathcal{U}$ in Eqs. (2.3) and (2.4), is given by the time-ordered product,

$$
U\left(t, t^{\prime}\right)=T\left[\exp \left(-i \int_{t^{\prime}}^{t} H\left(t_{1}\right) d t_{1}\right)\right],
$$

with the time-dependent LF Hamiltonian (2.9) in the exponent. An exact evaluation of the double-time Green's function (2.11) rests on the exact calculation of the propagator $U\left(t, t^{\prime}\right)$, which is not possible in general. On the other hand, the only time dependence in $H(t)$ is through the coupling function $g(t)$, which makes $U\left(t, t^{\prime}\right)$ quite close to the propagator $e^{-i\left(t-t^{\prime}\right) H}$ of the time-independent Hamiltonian, at least for sufficiently slowly varying $g(t)$ and over a sufficiently small time interval $t-t^{\prime}$. It suggests that there may be a scheme to systematically expand the propagator $U\left(t, t^{\prime}\right)$ in powers of the derivative $g^{\prime}(t)$. Indeed we have found such a scheme as outlined below.

\section{B. Derivation of the adiabatic Green's function}

One can rewrite $U\left(t, t^{\prime}\right)$ in Eq. (2.12) as a product over discrete time slices in the spirit of Feynman,

$$
U\left(t, t^{\prime}\right)=e^{-i \Delta t H(t)} \cdots e^{-i \Delta t H\left(t_{i}\right)} \cdots e^{-i \Delta t H\left(t^{\prime}\right)},
$$

and note that any given $e^{-i \Delta t H\left(t_{i}\right)}$ can be diagonalized exactly by the time-dependent unitary operator, $\mathcal{U}\left(t_{i}\right)$ :

$$
\begin{aligned}
\bar{H}\left(t_{i}\right) & =\mathcal{U}^{\dagger}\left(t_{i}\right) H\left(t_{i}\right) \mathcal{U}\left(t_{i}\right)=\bar{\varepsilon}\left(t_{i}\right) c^{\dagger} c+\omega_{0} a^{\dagger} a, \\
\mathcal{U}\left(t_{i}\right) & =e^{g\left(t_{i}\right) c^{\dagger} c\left(a-a^{\dagger}\right)}, \\
\bar{\varepsilon}\left(t_{i}\right) & =\varepsilon-g\left(t_{i}\right)^{2} \omega_{0} .
\end{aligned}
$$

The replacement

$$
e^{-i \Delta t H\left(t_{i}\right)} \rightarrow \mathcal{U}\left(t_{i}\right) e^{-i \Delta t \bar{H}\left(t_{i}\right)} \mathcal{U}^{\dagger}\left(t_{i}\right)
$$

in Eq. (2.13) gives another expression of the propagator

$$
\begin{aligned}
U\left(t, t^{\prime}\right)= & \left(\mathcal{U}(t) e^{-i \Delta t \bar{H}(t)} \mathcal{U}^{\dagger}(t)\right) \cdots\left(\mathcal{U}\left(t_{i}\right) e^{-i \Delta t \bar{H}\left(t_{i}\right)} \mathcal{U}^{\dagger}\left(t_{i}\right)\right) \\
& \cdots\left(\mathcal{U}\left(t^{\prime}\right) e^{-i \Delta t \bar{H}\left(t^{\prime}\right)} \mathcal{U}^{\dagger}\left(t^{\prime}\right)\right)
\end{aligned}
$$

The essential idea here is the use of "instantaneous unitary operator" $\mathcal{U}\left(t_{i}\right)$ with which to diagonalize the evolution operator $e^{-i \Delta t H\left(t_{i}\right)}$ locally in time.

Another way to organize the product (2.16) is

$$
\cdots e^{-i \Delta t \bar{H}\left(t_{i+1}\right)}\left[\mathcal{U}^{\dagger}\left(t_{i+1}\right) \mathcal{U}\left(t_{i}\right)\right] e^{-i \Delta t \bar{H}\left(t_{i}\right)} \cdots .
$$

Due to the fact that unitary operators $\mathcal{U}\left(t_{i}\right)$ at different time slices do not commute, there is a factor $\mathcal{U}^{\dagger}\left(t_{i+1}\right) \mathcal{U}\left(t_{i}\right)$ sandwiched between a pair of adjacent exponentials $e^{-i \Delta t \bar{H}\left(t_{i+1}\right)}$ and $e^{-i \Delta t \bar{H}\left(t_{i}\right)}$ in the product (2.16). Since the time difference $t_{i+1}-t_{i}=\Delta t$ is by assumption very small, one can ignore the 
small noncommuting factor of order $(\Delta t)^{2}$ and combine the product $\mathcal{U}^{\dagger}\left(t_{i+1}\right) \mathcal{U}\left(t_{i}\right)$ as [31]

$$
\mathcal{U}^{\dagger}\left(t_{i+1}\right) \mathcal{U}\left(t_{i}\right) \approx e^{-g^{\prime}\left(t_{i}\right) \Delta t c^{\dagger} c\left(a-a^{\dagger}\right)} .
$$

In other words, the exact propagator $U\left(t, t^{\prime}\right)$ is obtained from path-ordered exponential of the new effective Hamiltonian

$$
\begin{aligned}
I(t) & =\bar{H}(t)-i g^{\prime}(t) c^{\dagger} c\left(a-a^{\dagger}\right), \\
\bar{H}(t) & =\bar{\varepsilon}(t) c^{\dagger} c+\omega_{0} a^{\dagger} a,
\end{aligned}
$$

as

$$
\begin{aligned}
U\left(t, t^{\prime}\right) & =\mathcal{U}(t) \bar{U}\left(t, t^{\prime}\right) \mathcal{U}^{\dagger}\left(t^{\prime}\right) . \\
\bar{U}\left(t, t^{\prime}\right) & =T\left[\exp \left(-i \int_{t^{\prime}}^{t} I\left(t_{1}\right) d t_{1}\right)\right] .
\end{aligned}
$$

The new Hamiltonian $I(t)$ contains the first derivative of the coupling, $g^{\prime}(t)$, not $g(t)$ itself, and much more amenable to perturbative treatment in powers of the small function $g^{\prime}(t)$. Another way to view $I(t)$ is as a time-dependent unitary rotation

$$
I(t)=\mathcal{U}^{\dagger}(t) H(t) \mathcal{U}(t)-i \mathcal{U}^{\dagger}(t) \partial_{t} \mathcal{U}(t)
$$

which yields the same expression as Eq. (2.19). Note that Eq. (2.20) is still an exact writing of the propagator.

The next stage of evaluation involves some perturbative scheme, under the adiabaticity assumption. We have developed the interaction picture scheme to write down the propagator as a power series in $g^{\prime}(t)$. Details are involved and can be found in Appendix A. Here, we just quote the zeroth-order result for the Green's function:

$$
\begin{aligned}
G^{>,(0)}\left(t, t^{\prime}\right)= & -i e^{-i \int_{t^{\prime}}^{t} d t_{1}\left[\bar{\varepsilon}\left(t_{1}\right)-g^{\prime}\left(t_{1}\right)^{2} / \omega_{0}\right]}\left\langle\alpha\left|X(t) c c^{\dagger} X^{\dagger}\left(t^{\prime}\right)\right| \alpha\right\rangle, \\
\left\langle\alpha\left|X(t) c c^{\dagger} X^{\dagger}\left(t^{\prime}\right)\right| \alpha\right\rangle= & \exp \left[g(t) g\left(t^{\prime}\right) e^{-i \omega_{0}\left(t-t^{\prime}\right)}-\frac{1}{2}\left(g(t)^{2}+g\left(t^{\prime}\right)^{2}\right)\right] \\
& \times \exp \left[\alpha e^{i \omega_{0} t_{0}}\left(g(t) e^{-i \omega_{0} t}-g\left(t^{\prime}\right) e^{-i \omega_{0} t^{\prime}}\right)\right] \exp \left[-\alpha^{*} e^{-i \omega_{0} t_{0}}\left(g(t) e^{i \omega_{0} t}-g\left(t^{\prime}\right) e^{i \omega_{0} t^{\prime}}\right)\right] .
\end{aligned}
$$

We label it the adiabatic Green's function for an obvious reason. While it is difficult to compare the validity of this Green's function against an exact one for general $g(t)$, our calculation in the following section confirms that corrections up to the second order make negligible difference to the zeroth-order one given above. Although a vast amount of literature was devoted to the study of time-dependent and transient dynamics in quantum models, here the Green's function valid in the adiabatic limit is explicitly written down.

\section{TRANSIENT BEHAVIOR OF THE GREEN'S FUNCTION}

According to Ref. [28], the time-resolved photoemission spectroscopy (TR-PES) intensity at the binding energy $\omega$, $P\left(t_{p}, \omega\right)$, is obtained from the formula

$$
\begin{aligned}
P\left(t_{p}, \omega\right) \approx & -i \int_{-\infty}^{\infty} d t_{2} \int_{-\infty}^{\infty} d t_{1} s\left(t_{1}-t_{p}\right) s\left(t_{2}-t_{p}\right) \\
& \times e^{i \omega\left(t_{1}-t_{2}\right)} G^{<}\left(t_{1}, t_{2}\right) .
\end{aligned}
$$

The probe pulse shape function $s\left(t-t_{p}\right)$ is determined by the specific experimental setup. We choose the step-function profile

$$
s\left(t-t_{p}\right)=\theta\left(t-t_{p}\right)-\theta\left(t-\sigma_{\mathrm{pr}}-t_{p}\right)
$$

that corresponds to the probe pulse duration $t_{p}<t<t_{p}+\sigma_{\mathrm{pr}}$. $P\left(t_{p}, \omega\right)$ records the total accumulated photocurrent over the pulse duration $\sigma_{\mathrm{pr}}$ which started at time $t_{p}$. Reference [32] showed that the resolution of TR-PES $\sigma_{\text {res }}$ is proportional to the inverse of temporal width of probe pulse: $\sigma_{\mathrm{pr}} \sim 1 / \sigma_{\text {res }}$. Since we want to make $\sigma_{\text {res }} \ll \omega_{0}$, we set $\sigma_{\text {pr }}=10 \tau_{0}$, where $\tau_{0}=$ $2 \pi / \omega_{0}$. The nonequilibrium system itself is prepared at time $t_{0}$ which is set at the far past. Throughout the time evolution $t_{0}<$ $t<t_{p}+\sigma_{\mathrm{pr}}$ the system is governed by the time-dependent LF Hamiltonian $H(t)$.

The lesser Green's function $G^{<}\left(t, t^{\prime}\right)$ in the intensity formula

$$
G^{<}\left(t, t^{\prime}\right)=i \operatorname{Tr}\left[c^{\dagger}\left(t^{\prime}\right) c(t) \rho\right],
$$

which is different from the one analyzed in the previous section, can be solved with the same method. There is a certain degree of freedom in choosing the initial state $|\psi\rangle$ and the initial density matrix $\rho=|\psi\rangle\langle\psi|$. Our choice for $|\psi\rangle$ is a product of the boson coherent state and a one-electron state, hybridized by the unitary operator $\mathcal{U}$ :

$$
|\psi\rangle=\mathcal{U}\left(c^{\dagger}|\alpha\rangle\right)=e^{(g / 2)\left(\alpha-\alpha^{*}\right)} c^{\dagger}|\alpha-g\rangle .
$$

Using $\rho=|\psi\rangle\langle\psi|$,

$$
G^{<}\left(t, t^{\prime}\right)=i\left\langle\alpha-g\left|c c^{\dagger}\left(t^{\prime}\right) c(t) c^{\dagger}\right| \alpha-g\right\rangle .
$$

Unlike the greater Green's function case, we set occupied fermion state in the initial state since empty fermion state gives zero to the lesser Green's function. Evaluating the lesser Green's function yields

$$
\begin{aligned}
G^{<,(0)}\left(t, t^{\prime}\right)= & i \exp \left[-i \int_{t^{\prime}}^{t} d t_{1}\left(\bar{\varepsilon}\left(t_{1}\right)-\frac{g^{\prime}\left(t_{1}\right)^{2}}{\omega_{0}}\right)\right] \\
& \times \exp \left[g(t) g\left(t^{\prime}\right) e^{i \omega_{0}\left(t-t^{\prime}\right)}-\frac{1}{2}\left(g(t)^{2}+g\left(t^{\prime}\right)^{2}\right)\right] \\
& \times \exp \left[\alpha e^{i \omega_{0} t_{0}}\left(g(t) e^{-i \omega_{0} t}-g\left(t^{\prime}\right) e^{-i \omega_{0} t^{\prime}}\right)\right] \\
& \times \exp \left[-\alpha^{*} e^{-i \omega_{0} t_{0}}\left(g(t) e^{i \omega_{0} t}-g\left(t^{\prime}\right) e^{i \omega_{0} t^{\prime}}\right)\right] .
\end{aligned}
$$

We also obtained the first and second corrections for lesser Green's functions, $G^{<,(1)}\left(t, t^{\prime}\right)$ and $G^{<,(2)}\left(t, t^{\prime}\right)$, as reproduced in Appendix B. The total lesser Green's function up to second 
order in the derivative $g^{\prime}(t)$ is the sum,

$$
G^{<}\left(t, t^{\prime}\right)=G^{<,(0)}\left(t, t^{\prime}\right)+G^{<,(1)}\left(t, t^{\prime}\right)+G^{<,(2)}\left(t, t^{\prime}\right) .
$$

An explicit numerical evaluation finds negligible contributions to the photocurrent from higher-order Green's functions $G^{<,(1)}$ and $G^{<,(2)}$, making the zeroth-order Green's function we derived in Eq. (3.6) essentially exact for the time-varying coupling $g(t)$. The conditions for their validity are that the typical variation in $g(t)$ occurs over a time scale much longer than the oscillator period, $\left|g^{\prime}(t) \tau_{0} / g(t)\right| \ll 1$, and that $g^{\prime}(t)$ itself varies little over one period $\tau_{0}$. The second assumption, however, is natural in light of the first. Given that the typical pump laser in use today operates at the subvisible range, $\omega_{0} \sim 10^{13} \mathrm{~Hz}$, this is a rather comfortable assumption to be made.

How will the photocurrent intensity $P(t, \omega)$ evolve over time as the electron-boson coupling $g(t)$ is adiabatically turned off to zero? To explore this, we proceed to the numerical evaluation of $P(t, \omega)$ using the profile

$$
g(t)=\frac{g}{e^{t / T_{g}}+1}
$$

for the coupling function $g(t)$. Here, we set $T_{g}=3.2 \tau_{0}$. The adiabatic condition

$$
\left|\frac{g^{\prime}(t) \tau_{0}}{g(t)}\right|=\frac{\tau_{0}}{T_{g}} \frac{1}{e^{t / T_{g}}+1} \lesssim \frac{\tau_{0}}{2 T_{g}} \ll 1
$$

is fulfilled at all times $|t| \lesssim T_{g}$. The observation time $t$ extends from $-45 \tau_{0}$ up to $45 \tau_{0}$ in our calculation. The initial preparation time $t_{0}$ is set further back at $t_{0}=-130 \tau_{0}$. For the parameters of the model we choose $g=0.5$ and $\varepsilon=\omega_{0}$, which gives the renormalized energy $\bar{\varepsilon}=0.75 \omega_{0}$. The lesser Green's function can be obtained numerically for various choices of the coherent state $\alpha$. The phase angle in $\alpha$ can be absorbed since it always appears as the product $\alpha e^{i \omega_{0} t_{0}}$ in the Green's function [see Eq. (3.1)].

From the calculations, it turns out the two higher-order Green's functions in orders of $g^{\prime}$ and $\left(g^{\prime}\right)^{2}$ make negligible contributions to the photocurrent $P(t, \omega)$ for the $g(t)$ chosen in Eq. (3.8). Writing the photocurrent intensity $P^{(i)}(t, \omega)$ with superscript $i=\{1,2\}$ as arising from order $g^{\prime}$ and $\left(g^{\prime}\right)^{2}$ contributions, we found

$$
\frac{\left|\int d \omega P^{(i)}(t, \omega)\right|}{\left|\int d \omega P^{(0)}(t, \omega)\right|} \approx 10^{-7},
$$

which is negligible, for the entire $t$ in our calculation. We can understand this from the structure of the zeroth-order Green's function shown in Eq. (3.6), and comparing it to higher-order Green's functions shown in Appendix B. Higherorder Green's functions are essentially $G^{<,(0)}$ multiplied by the fast oscillating functions $e^{ \pm i \omega_{0} t}$ and vanishes easily upon integrating over many cycles. In this regard the adiabatic method we developed to obtain the two-time Green's function for the time-dependent LF Hamiltonian is already exact at the zeroth order.

Figure 1 shows the photocurrent $P(t, \omega)$ at several times $t$ throughout the adiabatic turn-off of the coupling $g(t)$. Several sidebands, present at times long before the adiabatic turn-off process began, have their frequencies shifted by $\sim g^{2} \omega_{0}=0.25 \omega_{0}$ as $g$ diminishes to zero. Their intensities (a)

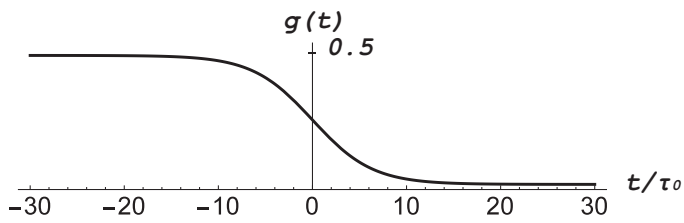

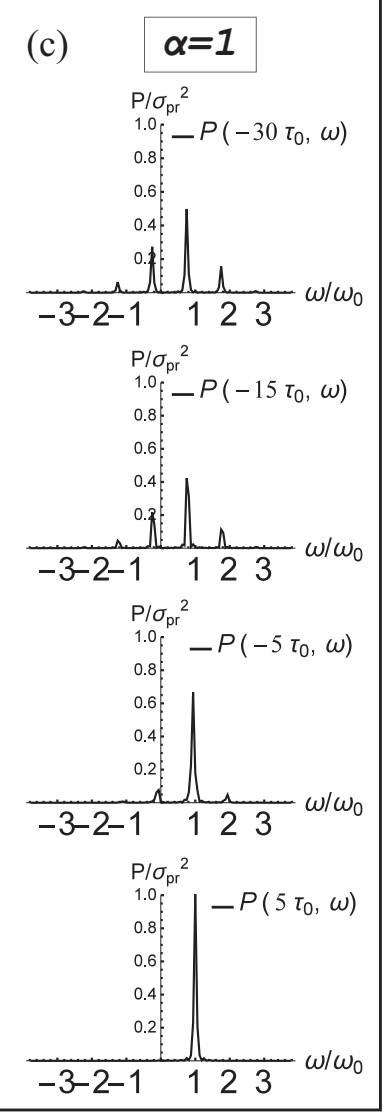

FIG. 1. (a) Profile of the coupling $g(t)$ over time. Frequency resolved photocurrent intensity $P(t, \omega)$ at various times for the coherent state amplitudes (b) $\alpha=0$ and (c) $\alpha=1$. Fully developed sideband feature at $\omega=\bar{\varepsilon}+$ (integer) $\omega_{0}$ shown at $t=-30 \tau_{0}$ starts to slide over to the higher frequency for times past $t=-15 \tau_{0}$ where $g^{\prime}(t)$ also starts to vary. The sideband intensities vanish at $t=5 \tau_{0}$.

diminish over time. The main peak at the energy $\bar{\varepsilon}(t)$ also slides in frequency by $g^{2} \omega_{0}=0.25 \omega_{0}$ with its intensity growing over time. Even for the $\alpha=0$ case, we can see that the sidebands emerge at $\omega=\bar{\varepsilon}-n \omega_{0}(n>0)$. These sidebands for $\alpha=0$ are coming from the terms proportional to the $g^{2}$ in Eq. (3.6).

It is notable that we obtain the diminishing sidebands feature even without manifestly introducing the dissipation mechanism, such as the bosonic bath, explicitly [17]. In an adiabatic evolution of the quantum system such as the expanding potential well, the instantaneous energy of the system smoothly follows the ground state value of the instantaneous Hamiltonian. As the wall expands the energy also diminishes, but this is done without an explicit dissipation mechanism. The same phenomenon is happening in our Green's function treatment of the adiabatic evolution. 


\section{A. Transient behavior in the semiclassical limit}

The boson field is treated as a quantized oscillator in our approach to transient dynamics. In this subsection, we ask what happens if the boson field is treated semiclassically, and the relevant Hilbert space is that of fermions only. The semiclassical limit of the time-dependent LF Hamiltonian is obtained by going to the interaction picture, $a \rightarrow a e^{-i \omega_{0}\left(t-t_{0}\right)}$,

$$
\begin{aligned}
\hat{H}_{\mathrm{LF}}(t) & =e^{i \int_{t_{0}}^{t} d t_{1} H_{b}} H_{\mathrm{LF}}(t) e^{-i \int_{t_{0}}^{t} d t_{1} H_{b}}-\omega_{0} a^{\dagger} a \\
& =\varepsilon c^{\dagger} c+g(t) \omega_{0} c^{\dagger} c\left(a e^{-i \omega_{0}\left(t-t_{0}\right)}+a^{\dagger} e^{i \omega_{0}\left(t-t_{0}\right)}\right),
\end{aligned}
$$

and then replacing $a$ by its average $\langle a\rangle=\alpha$, assuming a coherent state of the boson,

$$
H_{\mathrm{LF}}^{c l} .(t)=\varepsilon c^{\dagger} c+2 g(t) \omega_{0} \alpha \cos \left[\omega_{0}\left(t-t_{0}\right)\right] c^{\dagger} c .
$$

The lesser Green's function for the semiclassical, timedependent LF model is still of the form,

$$
G^{<}\left(t, t^{\prime}\right)=i \operatorname{Tr}\left[\rho\left(t_{0}\right) c^{\dagger}\left(t^{\prime}\right) c(t)\right] .
$$

The Hilbert space is now confined to the two-level fermion states only, and the density matrix $\rho\left(t_{0}\right)=|\psi\rangle\langle\psi|$ consists of the one-fermion state $|\psi\rangle=c^{\dagger}|0\rangle$. The lesser Green's function for arbitrary coupling $g(t)$ becomes

$$
\begin{aligned}
G^{<}\left(t, t^{\prime}\right)= & i e^{-i \varepsilon\left(t-t^{\prime}\right)-i \int_{t^{\prime}}^{t} d t_{1} 2 g\left(t_{1}\right) \omega_{0} \alpha \cos \omega_{0}\left(t_{1}-t_{0}\right)} \\
\approx & i e^{-i \varepsilon\left(t-t^{\prime}\right)} \exp \left[\alpha e^{-i \omega_{0} t_{0}}\left(g(t) e^{i \omega_{0} t}-g\left(t^{\prime}\right) e^{i \omega_{0} t^{\prime}}\right)\right] \\
& \times \exp \left[-\alpha e^{i \omega_{0} t_{0}}\left(g(t) e^{-i \omega_{0} t}-g\left(t^{\prime}\right) e^{-i \omega_{0} t^{\prime}}\right)\right] .
\end{aligned}
$$

In the last line we have ignored terms proportional to $g^{\prime}(t)$, as allowed by the adiabatic assumption Eq. (2.10). One can easily notice that Eq. (3.14) can be recovered by erasing the terms proportional to the $g^{2}$ in the arguments of exponentials in Eq. (3.6).

From the semiclassical, time-dependent Green's function (3.14) we obtain the photocurrent shown in Fig. 2. We have used the identical profile for the coupling function $g(t)$ as in the earlier, quantum-mechanical LF model [Eq. (3.8)] with $g=0.5$. We set the other parameters $\alpha=1$ and $\varepsilon=0.75 \omega_{0}$. Again, in the photocurrent calculation the probe beam starts at $t_{p}=-45 \tau_{0}$ and observation time ends at $t_{p}=45 \tau$. The initial preparation time $t_{0}$ is set at $t_{0}=-130 \tau_{0}$. Initially, as shown in Fig. 2(a), there are well-developed sideband peaks in the semiclassical photocurrent $P(t, \omega)$ as well. As one turns $g(t)$ off the weights at sideband energies diminish and only the weight at the bare energy $\varepsilon$ grows monotonically.

A number of subtle differences exists between semiclassical and quantum calculations of the photocurrent profile. First, since there is no renormalization of the bare electron level $\varepsilon$ in the semiclassical limit, there cannot exist the sliding over feature of the peaks of photocurrent intensities. Next, the profile $P\left(t_{p}, \omega\right)$ in the semiclassical calculation remains completely symmetric about $\omega=\varepsilon$ at all times $t_{p}$ since there are no spontaneous emission of boson in the classical limit. The sidebands of semiclassical calculation are fully due to the terms proportional to $\alpha$ in Eq. (3.14). Even with these subtle differences, it is notable that the semiclassical Green's function (a)

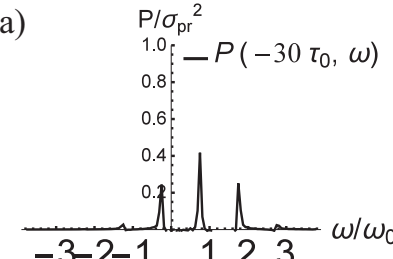

(c)

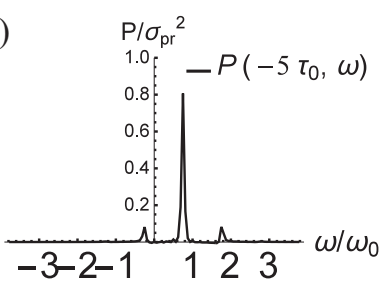

(b)

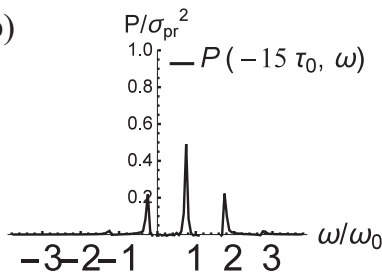

(d)

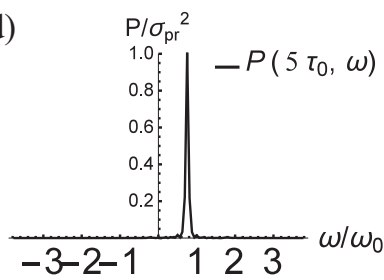

FIG. 2. Frequency-resolved photocurrent intensity $P(t, \omega)$ at various times for semiclassical LF model with $\alpha=1$ and $\varepsilon=$ $0.75 \omega_{0}$. Fully developed sidebands at $\omega=\varepsilon+$ (integer) $\omega_{0}$ are shown in (a). For the classical case, the intensities of sidebands have bilateral symmetry with respect to the bare electron energy $\varepsilon$. In (b)-(d), the intensity corresponding to the bare electron energy monochromatically increases as time evolves while other sideband intensities monotonically decrease.

is recovered in the large $\alpha$ limit of Eq. (3.6). This fact is consistent with the idea of considering boson field classically in the limit of large number of boson $N=|\alpha|^{2}$. Since the calculation for the semiclassical calculation is straightforward, the fact that the Green's function in Eq. (3.6) is recovered by the semiclassical Green's function supports that our method is reasonable.

\section{B. Time-dependent spin-boson model}

Techniques we developed to address the transient phenomena in the Lang-Firsov model with time-dependent coupling can be applied, with a little modification, to another well-known and popular spin-boson (SB) model describing the two-level system interacting with the bosonic field:

$$
H_{\mathrm{SB}}=\frac{\varepsilon}{2} \sigma_{z}+\frac{\Delta}{2} \sigma_{x}+\omega_{0} a^{\dagger} a+\frac{g}{2} \omega_{0} \sigma_{z}\left(a+a^{\dagger}\right) .
$$

This model for $\Delta=0$ is none other than the Lang-Firsov Hamiltonian by replacing $\sigma^{+} \rightarrow c^{\dagger}, \sigma^{-} \rightarrow c$, and $\sigma^{z}=$ $2 c^{\dagger} c-1$. The transition term $(\Delta / 2) \sigma_{x}$ between two energy levels does not have a fermion analog as it corresponds to single fermion annihilation and creation processes $\sim \Delta\left(c^{\dagger}+c\right)$

Applying the unitary operator $\mathcal{U}=e^{g \sigma_{z}\left(a-a^{\dagger}\right)}$ gives

$$
\begin{aligned}
\bar{H}_{\mathrm{SB}} & =\mathcal{U}^{\dagger} H_{\mathrm{SB}} \mathcal{U} \\
& =\frac{\varepsilon}{2} \sigma_{z}-\frac{g^{2}}{4} \omega_{0}+\omega_{0} a^{\dagger} a+\frac{\Delta}{2}\left(X^{\dagger} \sigma^{+}+X \sigma^{-}\right),
\end{aligned}
$$

where $X=e^{g\left(a-a^{\dagger}\right)}$. The interaction term $(g / 2) \omega_{0} \sigma_{z}\left(a+a^{\dagger}\right)$ is gone, but there is a residual interaction of order $\Delta$ in the 
transformed Hamiltonian $\bar{H}_{\mathrm{SB}}$. It turns out to be exceedingly difficult to keep both the time dependence of the coupling $g(t)$ and the residual interaction of order $\Delta$ in calculating the adiabatic Green's function. From now on we drop the $\Delta$ piece in the above and generalize the $\Delta=0$ spin-boson Hamiltonian to the time-dependent one:

$$
H_{\mathrm{SB}}(t)=\frac{\varepsilon}{2} \sigma_{z}+\omega_{0} a^{\dagger} a+\frac{1}{2} g(t) \omega_{0} \sigma_{z}\left(a+a^{\dagger}\right) .
$$

We assume that $g(t)$ is a slowly varying function in time and define the lesser Green's function for the SB Hamiltonian as

$$
G^{<}\left(t, t^{\prime}\right)=i \operatorname{Tr}\left[\rho\left(t_{0}\right) \sigma^{+}\left(t^{\prime}\right) \sigma^{-}(t)\right],
$$

where $\sigma^{ \pm}=\left(\sigma_{x} \pm i \sigma_{y}\right) / 2$. Choosing the initial state density matrix $\rho\left(t_{0}\right)=|\psi\rangle\langle\psi|$, where $|\psi\rangle=|\uparrow, \alpha-g\rangle$, calculation of the lesser Green's function proceeds in direct analogy with the one for the LF model. We obtain

$$
\begin{aligned}
G^{<,(0)}\left(t, t^{\prime}\right)= & i e^{-i \varepsilon\left(t-t^{\prime}\right)}\left\langle\uparrow, \alpha\left|\sigma^{+} \hat{X}\left(t^{\prime}\right) \sigma^{-} \hat{X}(t)\right| \uparrow, \alpha\right\rangle \\
= & i e^{-i \varepsilon\left(t-t^{\prime}\right)} \exp \left[g(t) g\left(t^{\prime}\right) e^{i \omega_{0}\left(t-t^{\prime}\right)}-\frac{1}{2}\left(g(t)^{2}+g\left(t^{\prime}\right)^{2}\right)\right] \\
& \times \exp \left[\alpha e^{i \omega_{0} t_{0}}\left(g(t) e^{-i \omega_{0} t}-g\left(t^{\prime}\right) e^{-i \omega_{0} t^{\prime}}\right)\right] \exp \left[-\alpha^{*} e^{-i \omega_{0} t_{0}}\left(g(t) e^{i \omega_{0} t}-g\left(t^{\prime}\right) e^{i \omega_{0} t^{\prime}}\right)\right] .
\end{aligned}
$$

One can see this expression is almost identical to the zerothorder Green's function worked out in Eq. (3.6).

The transient behavior in the photocurrent intensity $P(t, \omega)$ is shown in Fig. 3. The adiabatic behavior of the photocurrent is showing the smooth decay of the sideband weights over time. In the $\mathrm{SB}$ model the bare energy level $\varepsilon$ does not renormalize; hence we do not observe any sliding over behavior in the adiabatic turn-off process that characterized the transient dynamics of the LF model.

\section{Dirac electrons coupled to quantized photons}

Although our goal is a simple one-finding solutions to the adiabatic generalization of exactly solvable models - the work we did here may have a nontrivial implication to a much more physical situation of current interest. This is the problem of two-dimensional (2D) Dirac electrons coupled to the intense (a)

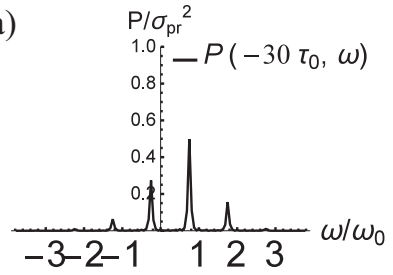

(c)

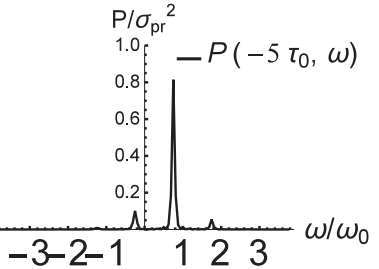

(b)

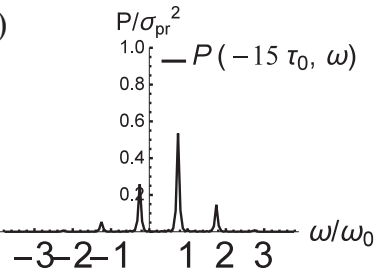

(d)

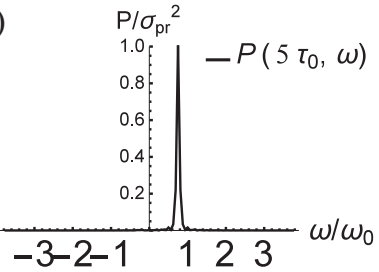

FIG. 3. Frequency-resolved zeroth-order contribution to the photocurrent intensity $P^{(0)}(t, \omega)$ at various times in the time-dependent spin-boson model with $\varepsilon=0.8 \omega_{0}$ and $g=0.5$. The coherent state amplitude is set to $\alpha=1$. (a) Fully developed sideband features exist as in the LF model. The sideband intensity is decreasing monotonically in (b)-(d) after $g(t)$ is turned off, as the intensity for the bare electron energy level increases and eventually saturates. We do not observe any "sliding over" behavior as in the LF model. laser as studied in several papers in recent years [29,30,33-36]. The Hamiltonian for 2D Dirac electrons coupled to the laser is given by

$$
H(t)=\int d \mathbf{r} \psi^{\dagger}(\mathbf{r})[v(-i \nabla+e \mathbf{A}(\mathbf{r}, t)) \times \boldsymbol{\sigma} \cdot \hat{\mathbf{y}}] \psi(\mathbf{r}),
$$

where $\sigma$ are the Pauli matrices, $\hat{\mathbf{y}}$ is normal to the 2D surface, and $\psi(\mathbf{r})=(u(\mathbf{r}), d(\mathbf{r}))^{\mathrm{T}}$ are the real-space versions of the spinup $u_{\mathbf{k}}$ and spin-down $d_{\mathbf{k}}$ operators. We have set $\hbar=1$. The vector potential for the laser light is written in the following second-quantized form:

$$
\mathbf{A}(\mathbf{r}, t)=\sum_{\mathbf{p}} \sqrt{\frac{1}{2 \epsilon_{0} \omega_{\mathbf{p}} V}}\left(\epsilon_{\mathbf{p}} a_{\mathbf{p}} e^{i \mathbf{p} \cdot \mathbf{r}-i \omega_{\mathbf{p}} t}+\text { H.c. }\right) .
$$

Here, $\epsilon_{0}$ is the dielectric constant, $\omega_{\mathbf{p}}$ is the frequency of laser at momentum $\mathbf{p}$, and $V$ is the volume of the box. We can choose a monochromatic frequency for the laser $\omega_{\mathbf{p}}=\omega_{0}$, and the perpendicular direction of incidence for which $\epsilon_{\mathbf{p}} \cdot \hat{\mathbf{y}}=0$. Keeping the $\mathbf{p}=\mathbf{0}$ component of the vector potential only gives

$$
\begin{aligned}
& H(t) \approx \sum_{\mathbf{k}} \psi_{\mathbf{k}}^{\dagger}\left[v\left(\mathbf{k}-e \mathbf{A}_{\mathbf{p}=\mathbf{0}}(t)\right) \times \boldsymbol{\sigma} \cdot \hat{\mathbf{y}}\right] \psi_{\mathbf{k}}, \\
& \mathbf{A}_{\mathbf{0}}(t)=\frac{g \omega_{0}}{e v}\left(\epsilon_{\mathbf{0}} a_{\mathbf{0}} e^{-i \omega_{0} t}+\epsilon_{\mathbf{0}}^{*} a_{\mathbf{0}}^{\dagger} e^{i \omega_{0} t}\right),
\end{aligned}
$$

where $g=e v / \sqrt{2 \epsilon_{0} \omega_{0}^{3} V}$. For the linear polarization of the incident laser we can choose $\epsilon_{\mathbf{0}}=(1,0,0)$, and the Hamiltonian becomes

$H(t)$

$$
=\sum_{\mathbf{k}} \psi_{\mathbf{k}}^{\dagger}\left[\left(-v k_{x}+g \omega_{0}\left(a_{\mathbf{0}} e^{-i \omega_{0} t}+a_{\mathbf{0}}^{\dagger} e^{i \omega_{0} t}\right)\right) \sigma_{z}+v k_{z} \sigma_{x}\right] \psi_{\mathbf{k}} .
$$

We then introduce the rotating wave approximation by taking the time-dependent unitary transformation

$$
H(t) \mapsto H^{\prime}(t)=U^{\dagger}(t) H(t) U(t)-i U^{\dagger}(t) \partial_{t} U(t),
$$


with the time-dependent unitary operator $U(t)=e^{i \omega_{0} t a_{0}^{\dagger} a_{0}}$. Fast-oscillating terms in $H^{\prime}(t)$ containing the time-dependent phase $e^{ \pm i \omega_{0} t}$ are dropped, and one can get the time-independent Hamiltonian:

$$
\begin{aligned}
H= & \sum_{\mathbf{k}} \psi_{\mathbf{k}}^{\dagger}\left[\left(-v k_{x}+g \omega_{0}\left(a_{\mathbf{0}}+a_{\mathbf{0}}^{\dagger}\right)\right) \sigma_{z}+v k_{z} \sigma_{x}\right] \psi_{\mathbf{k}} \\
& +\omega_{0} a_{\mathbf{0}}^{\dagger} a_{\mathbf{0}} .
\end{aligned}
$$

Despite the simple appearance of Eq. (3.25), there is difficulty in solving this problem due to the fact that electrons with different momenta $\mathbf{k}$ are all coupled to the single photon mode $\omega_{0}$ and thereby coupled with each other, much like in the single-impurity Kondo problem. However, if we could consider a slightly modified problem in which each electron at momentum $\mathbf{k}$ couples to its own photon mode independently, the problem posed by Eq. (3.25) becomes

$$
\begin{aligned}
H= & \sum_{\mathbf{k}} \psi_{\mathbf{k}}^{\dagger}\left[\left(\frac{\varepsilon_{\mathbf{k}}}{2}+g \omega_{0}\left(a_{\mathbf{k}}+a_{\mathbf{k}}^{\dagger}\right)\right) \sigma_{z}+\frac{\Delta_{\mathbf{k}}}{2} \sigma_{x}\right] \psi_{\mathbf{k}} \\
& +\omega_{0} \sum_{\mathbf{k}} a_{\mathbf{k}}^{\dagger} a_{\mathbf{k}} .
\end{aligned}
$$

Here, $\varepsilon_{\mathbf{k}}=-2 v k_{x}$ and $\Delta_{\mathbf{k}}=2 v k_{z}$. Note now that the Hilbert spaces with different electron momenta $\mathbf{k}$ are decoupled, enabling us to reduce the problem to that of a single-electron coupled to the quantized photon. One can see that each momentum sector of this Hamiltonian is a realization of the well-known spin-boson model, widely used in theories of quantum optics, quantum dissipation, quantum computation, and circuit quantum electrodynamics [22-24]. In the limit of $\Delta_{\mathbf{k}} \rightarrow 0$, i.e., $k_{x} \rightarrow 0$, the SB model reduces to the LF model. In this regard, one can connect the 2D Dirac system coupled with the quantized laser field to the LF model. The Floquet theory does not work for the 2D Dirac model coupled to the quantized radiation field. The quenching of the laser pulse, which is a critical aspect in the time-resolved ARPES experiments, can be mimicked by the time dependence of the coupling $g=g(t)$. Although this independent photon coupling is a crude approximation, we believe that our analytical treatment of the LF and SB problem can serve as the first step towards the challenging goal of solving the 2D Dirac problem interacting with quantized light field.

\section{DISCUSSION}

Understanding the transient dynamics of electron-boson coupled system is of growing theoretical importance as pump-probe type experiments get refined at a rapid pace and begin to demonstrate fascinating phenomena [29,37,38]. In this paper we attempt to give theoretical foundation to addressing the question, "How do the electronic sidebands die out after the pump laser is turned off?", by solving in a quasiexact manner the time-dependent versions of the Lang-Firsov and spin-boson Hamiltonians. Our calculation successfully demonstrates the gradual decay of sidebands after the pump has been decoupled from the electronic system. Existence of the dissipative environment is not a necessary condition to observe the decay in the adiabatic limit as opposed to the previous study $[7,8,11,16-19]$.
A key theoretical idea allowing us to obtain the nonequilibrium Green's function is the introduction of "instantaneous basis" of unitary operators $U(t)$, that diagonalizes the Hamiltonian $H(t)$ exactly through the rotation $U^{\dagger}(t) H(t) U(t)$. The small discrepancy in the unitary operators at infinitesimally separated times $U(t+\Delta t) U^{\dagger}(t)$ can be treated perturbatively provided the time evolution of the parameter $g(t)$ in the Hamiltonian is slow in comparison to the characteristic oscillation period $\tau_{0}$. Readers are alerted to the similarity of our idea to Berry's derivation of the geometric phase, which he accomplished by solving the time-dependent Hamiltonian in the instantaneous basis of eigenstates. Berry's adiabatic solution of the wave function [20] has an analog in our approach as the zeroth-order Green's function. Corrections to the adiabatic Green's function can be generated by diligent application of the perturbative quantum field theory technique. In the case of time-dependent Lang-Firsov model those perturbative corrections are proved to have negligible impact on the time-dependent photocurrent intensity profile.

A related theoretical investigation of time-dependent electron dynamics in the Holstein model in the context of pumpprobe ARPES can be found in Ref. [32]. In their study the time-dependent part is the classical radiation field represented as the Peierls substitution of the momentum. While many aspects of the relaxation phenomena were discussed in that paper, sideband features and their demise after the quench were not. Also noteworthy is that the method employed in this work is not the prototypical Keldysh technique. Our approach is one of directly evaluating the two-time Green's function as accurately as possible, with the results shown in Eqs. (3.6) and (3.19) in essentially exact forms. A number of works studied the nonequilibrium phenomena in the context of a quantum dot coupled to external leads [8-16,18,19]. The dot Hamiltonian is akin to the Lang-Firsov model we study in this paper. We propose that the adiabatic Green's function derived here can be adopted to the more physical situation of a quantum dot under nonequilibrium and time-dependent conditions. As discussed in Sec. III C, our Green's function approach developed here can also shed some light on the more realistic problem about Dirac fermions coupled to quantized photons.

\section{ACKNOWLEDGMENTS}

Y.-T.O. was supported by a Global Ph.D. Fellowship Program through the National Research Foundation of Korea (NRF) funded by the Ministry of Education (No. NRF2014H1A2A1018320). J.H.H. acknowledges many insightful discussions on time-dependent phenomena with Patrick Lee and thanks him for hospitality during his sabbatical leave in 2014. Part of the motivation for this work came from discussions with Nuh Gedik.

\section{APPENDIX A: COMPLETION OF DERIVATION OF GREEN'S FUNCTION $G^{>}\left(t, t^{\prime}\right)$ FOR TIME-DEPENDENT LANG-FIRSOV MODEL}

In this appendix we introduce our trick to calculate further propagator $\bar{U}\left(t, t^{\prime}\right)$ in Eq. (2.20) to complete the calculation of the Green's function $G^{>}\left(t, t^{\prime}\right)$. Since we assumed $g^{\prime}(t)$ small, we can now treat the interaction as perturbation. In 
the interaction picture, the propagator becomes

$$
\bar{U}\left(t, t^{\prime}\right)=e^{-i \int_{t_{0}}^{t} d t_{1} \bar{H}\left(t_{1}\right)} \mathcal{S}\left(t, t^{\prime}\right) e^{i \int_{t_{0}}^{t} d t_{1} \bar{H}\left(t_{1}\right)},
$$

in which each operator $A(t)$ becomes

$$
\hat{A}(t)=e^{i \int_{t_{0}}^{t} d t_{1} \bar{H}\left(t_{1}\right)} A(t) e^{-i \int_{t_{0}}^{t} d t_{1} \bar{H}\left(t_{1}\right)} .
$$

There is no need to time order the exponential $\exp \left[-i \int \bar{H}\left(t_{1}\right) d t_{1}\right]$ since operators at different times now commute: $\left[\bar{H}\left(t_{1}\right), \bar{H}\left(t_{2}\right)\right]=0$. One can easily verify necessary properties such as

$$
\mathcal{S}\left(t, t^{\prime \prime}\right) \mathcal{S}\left(t^{\prime \prime}, t^{\prime}\right)=S\left(t, t^{\prime}\right), \mathcal{S}\left(t^{\prime}, t\right)=\mathcal{S}^{\dagger}\left(t, t^{\prime}\right) .
$$

It is a simple exercise to derive equations of motion

$$
\begin{aligned}
\partial_{t} \hat{a}(t) & =-i \omega_{0} \hat{a}(t), \quad \partial_{t} \hat{c}(t)=-i \bar{\varepsilon}(t) \hat{c}(t), \\
\partial_{t} \mathcal{S}\left(t, t^{\prime}\right) & =-c^{\dagger} c g^{\prime}(t)\left(\hat{a}(t)-\hat{a}^{\dagger}(t)\right) \mathcal{S}\left(t, t^{\prime}\right),
\end{aligned}
$$

and integrate them to obtain

$$
\begin{aligned}
& \hat{a}(t)=e^{-i \omega_{0}\left(t-t_{0}\right)} a, \quad \hat{c}(t)=e^{-i \int_{t_{0}}^{t} \bar{\varepsilon}\left(t_{1}\right) d t_{1}} c, \\
& \hat{X}(t)=\exp \left[g^{\prime}(t)\left(\hat{a}(t)-\hat{a}^{\dagger}(t)\right)\right],
\end{aligned}
$$

and

$$
\mathcal{S}\left(t, t^{\prime}\right)=T e^{\int_{t^{\prime}}^{t} d t_{1} g^{\prime}\left(t_{1}\right) c^{\dagger} c\left(\hat{a}^{\dagger}\left(t_{1}\right)-\hat{a}\left(t_{1}\right)\right)}
$$

in the interaction picture. The advantage of the interaction picture calculation is that the propagator $\mathcal{S}\left(t, t^{\prime}\right)$, Eq. (A4), depends only on the derivative $g^{\prime}\left(t_{1}\right)$ - a small quantity by assumption-and can be expanded as a power series. Expanding $\mathcal{S}\left(t, t^{\prime}\right)$ allows evaluation of the Green's function to successively higher orders of accuracy in $g^{\prime}(t)$.

Let's write the Green's function, Eq. (2.11), in the interaction basis. First we use Eq. (2.20) to express $G^{>}\left(t, t^{\prime}\right)$ as

$$
\begin{aligned}
G^{>}\left(t, t^{\prime}\right) & =i\left\langle\alpha\left|U\left(t_{0}, t\right) c U\left(t, t^{\prime}\right) c^{\dagger} U\left(t^{\prime}, t_{0}\right)\right| \alpha\right\rangle=i\left\langle\alpha\left|\mathcal{U}\left(t_{0}\right) \bar{U}\left(t_{0}, t\right) \mathcal{U}^{\dagger}(t) c \mathcal{U}(t) \bar{U}\left(t, t^{\prime}\right) \mathcal{U}^{\dagger}\left(t^{\prime}\right) c^{\dagger} \mathcal{U}\left(t^{\prime}\right) \bar{U}\left(t^{\prime}, t_{0}\right) \mathcal{U}^{\dagger}\left(t_{0}\right)\right| \alpha\right\rangle \\
& =i\left\langle\alpha\left|\bar{U}\left(t_{0}, t\right) c X(t) \bar{U}\left(t, t^{\prime}\right) c^{\dagger} X^{\dagger}\left(t^{\prime}\right) \bar{U}\left(t^{\prime}, t_{0}\right)\right| \alpha\right\rangle .
\end{aligned}
$$

The third line follows from $\mathcal{U}^{\dagger}(t) c \mathcal{U}(t)=c X(t), \mathcal{U}^{\dagger}(t) c^{\dagger} \mathcal{U}(t)=c^{\dagger} X^{\dagger}(t)$, where $X(t)=e^{g(t)\left(a-a^{\dagger}\right)}$. Furthermore we have $\mathcal{U}^{\dagger}\left(t_{0}\right)|\alpha\rangle=|\alpha\rangle$, since $c^{\dagger} c|\alpha\rangle=0$ due to the absence of fermions in the coherent state $|\alpha\rangle$. Now we go to the interaction picture and rewrite $G^{>}\left(t, t^{\prime}\right)$ as

$$
\begin{aligned}
G^{>}\left(t, t^{\prime}\right) & =i\left\langle\alpha\left|\bar{U}\left(t_{0}, t\right) c X(t) \bar{U}\left(t, t^{\prime}\right) c^{\dagger} X^{\dagger}\left(t^{\prime}\right) \bar{U}\left(t^{\prime}, t_{0}\right)\right| \alpha\right\rangle \\
& =i\left\langle\alpha\left|\mathcal{S}\left(t_{0}, t\right) e^{i \int_{t_{0}}^{t} \bar{H}\left(t_{1}\right) d t_{1}} c X(t) e^{-i \int_{t_{0}}^{t} \bar{H}\left(t_{1}\right) d t_{1}} \mathcal{S}\left(t, t^{\prime}\right) e^{i \int_{t_{0}}^{t^{\prime}} \bar{H}\left(t_{1}\right) d t_{1}} c^{\dagger} X^{\dagger}\left(t^{\prime}\right) e^{-i \int_{t_{0}}^{t^{\prime}} \bar{H}\left(t_{1}\right) d t_{1}} \mathcal{S}\left(t^{\prime}, t_{0}\right)\right| \alpha\right\rangle \\
& =i\left\langle\alpha\left|\mathcal{S}\left(t_{0}, t\right) \hat{c}(t) \hat{X}(t) \mathcal{S}\left(t, t^{\prime}\right) \hat{c}^{\dagger}\left(t^{\prime}\right) \hat{X}\left(t^{\prime}\right) \mathcal{S}\left(t^{\prime}, t_{0}\right)\right| \alpha\right\rangle=i e^{-i \int_{t^{\prime}}^{t} d t_{1} \bar{\varepsilon}\left(t_{1}\right)}\left\langle\alpha\left|\mathcal{S}\left(t_{0}, t\right) c \hat{X}(t) \mathcal{S}\left(t, t^{\prime}\right) c^{\dagger} \hat{X}^{\dagger}\left(t^{\prime}\right) \mathcal{S}\left(t^{\prime}, t_{0}\right)\right| \alpha\right\rangle
\end{aligned}
$$

This is the formally exact expression of the two-time Green's function for time-dependent LF model. Faithful evaluation of the Green's function becomes possible by systematically expanding $S\left(t, t^{\prime}\right)$ as a power series. By inspection of Eq. (A4) one concludes $\mathcal{S}\left(t, t^{\prime}\right)|\alpha\rangle=|\alpha\rangle$ for the zero-fermion state $|\alpha\rangle$, which means $G^{>}\left(t, t^{\prime}\right)$ further simplifies to

$$
G^{>}\left(t, t^{\prime}\right)=i e^{-i \int_{t^{\prime}}^{t} d t_{1} \bar{\varepsilon}\left(t_{1}\right)}\left\langle\alpha\left|c \hat{X}(t) \mathcal{S}\left(t, t^{\prime}\right) c^{\dagger} \hat{X}^{\dagger}\left(t^{\prime}\right)\right| \alpha\right\rangle
$$

The $\hat{X}\left(t^{\prime}\right)$ operator does not change the fermion number, while $c^{\dagger}$ raises it by one. When the next operator $\mathcal{S}\left(t, t^{\prime}\right)$ acts on the one-fermion state one can replace $c^{\dagger} c$ inside $S\left(t, t^{\prime}\right)$ by unity, so effectively,

$$
\mathcal{S}\left(t, t^{\prime}\right)=T\left[\exp \left(\int_{t^{\prime}}^{t} d t_{1} g^{\prime}\left(t_{1}\right)\left(\hat{a}^{\dagger}\left(t_{1}\right)-\hat{a}\left(t_{1}\right)\right)\right)\right] .
$$

The final technical hurdle in the Green's function evaluation is to develop a reliable expansion scheme for $\mathcal{S}\left(t, t^{\prime}\right)$ above. A simple Taylor expansion of the exponent won't work here-although that is how the typical diagrammatic calculation would proceed-due to the time-dependent function $g^{\prime}\left(t_{1}\right)$ in the integrand. The first step in this regard is to rewrite the propagator $\mathcal{S}\left(t, t^{\prime}\right)$ as a product of integrals over one oscillator period $\tau_{0}$ each,

$$
\mathcal{S}\left(t, t^{\prime}\right)=T \exp \left(-\int_{t^{\prime}+N \tau_{0}}^{t} d t_{1} g^{\prime}\left(t_{1}\right)\left(\hat{a}\left(t_{1}\right)-\hat{a}^{\dagger}\left(t_{1}\right)\right)\right) T \prod_{n=0}^{N} \exp \left(-\int_{t^{\prime}+(n-1) \tau_{0}}^{t^{\prime}+n \tau_{0}} d t_{1} g^{\prime}\left(t_{1}\right)\left(\hat{a}\left(t_{1}\right)-\hat{a}^{\dagger}\left(t_{1}\right)\right)\right) .
$$

It is understood that the last time slice $\left[t, t^{\prime}+N \tau_{0}\right]$ covers a fraction of the oscillator period $\tau_{0}$. For a particular time region $\left[t_{i}, t_{i}+\tau_{0}\right]$ we assume period $\tau_{0}$ to be small enough that the time ordering within this temporal region can be ignored. As a result it becomes possible to carry out the integral within each time slice; the second part of the right-hand side of Eq. (A9) becomes

$$
\begin{aligned}
& T\left[\exp \left(-\int_{t_{i}}^{t_{i}+\tau_{0}} d t_{1} g^{\prime}\left(t_{1}\right)\left(\hat{a}\left(t_{1}\right)-\hat{a}^{\dagger}\left(t_{1}\right)\right)\right)\right] \\
& \approx 1-\int_{t_{i}}^{t_{i}+\tau_{0}} d t_{1} g^{\prime}\left(t_{i}\right)\left(\left[a e^{-i \omega_{0}\left(t_{1}-t_{0}\right)}-a^{\dagger} e^{i \omega_{0}\left(t_{1}-t_{0}\right)}\right)+\int_{t_{i}}^{t_{i}+\tau_{0}} d t_{1} \int_{t_{i}}^{t_{1}} d t_{2} g^{\prime}\left(t_{i}\right)^{2}\left(a e^{-i \omega_{0}\left(t_{1}-t_{0}\right)}-a^{\dagger} e^{i \omega_{0}\left(t_{1}-t_{0}\right)}\right)\right. \\
& \quad \times\left(\left[a e^{-i \omega_{0}\left(t_{2}-t_{0}\right)}-a^{\dagger} e^{i \omega_{0}\left(t_{2}-t_{0}\right)}\right) \approx 1+i \frac{\left[g^{\prime}\left(t_{i}\right)\right]^{2}}{\omega_{0}} \tau_{0} \approx \exp \left(i \frac{\left[g^{\prime}\left(t_{i}\right)\right]^{2}}{\omega_{0}} \tau_{0}\right) .\right.
\end{aligned}
$$


First-order terms in $g^{\prime}(t)$ vanish from the integration over the full period of the harmonic oscillator, leaving a small, second-order correction from the integration. Since each term in the exponent is small, one can add them and express the result as an integral:

$$
T\left[\prod_{i=0}^{N} \exp \left(-\int_{t_{i}}^{t_{i}+\tau_{0}} d t_{1} g^{\prime}\left(t_{1}\right)\left(a\left(t_{1}\right)-\hat{a}^{\dagger}\left(t_{1}\right)\right)\right)\right] \approx \exp \left(i \int_{t^{\prime}}^{t} d t_{1} \frac{\left[g^{\prime}\left(t_{1}\right)\right]^{2}}{\omega_{0}}\right)
$$

The front exponential part of Eq. (A9) can be analyzed similarly,

$$
\begin{aligned}
& T\left[\exp \left(-\int_{t^{\prime}+N \tau_{0}}^{t} d t_{1} g^{\prime}\left(t_{1}\right)\left(\hat{a}\left(t_{1}\right)-\hat{a}\left(t_{1}\right)\right)\right)\right] \\
& \approx 1-\int_{t^{\prime}+N \tau_{0}}^{t} d t_{1} g^{\prime}(t)\left(a e^{-i \omega_{0}\left(t_{1}-t_{0}\right)}-a^{\dagger} e^{i \omega_{0}\left(t_{1}-t_{0}\right)}\right) \\
& \quad+\int_{t^{\prime}+N \tau_{0}}^{t} d t_{1} \int_{t^{\prime}+N \tau_{0}}^{t_{1}} d t_{1}\left[g^{\prime}(t)\right]^{2}\left(a e^{-i \omega_{0}\left(t_{1}-t_{0}\right)}-a^{\dagger} e^{i \omega_{0}\left(t_{1}-t_{0}\right)}\right)\left(a e^{-i \omega_{0}\left(t_{2}-t_{0}\right)}-a^{\dagger} e^{i \omega_{0}\left(t_{2}-t_{0}\right)}\right) \\
& \approx 1-i \frac{1}{\omega_{0}} g^{\prime}(t)\left(a e^{i \omega_{0} t_{0}}\left(e^{-i \omega_{0} t}-e^{-i \omega_{0} t^{\prime}}\right)+a^{\dagger} e^{-i \omega_{0} t_{0}}\left(e^{i \omega_{0} t}-e^{i \omega_{0} t^{\prime}}\right)\right) \\
& \quad-\frac{1}{2}\left(\frac{g^{\prime}(t)}{\omega_{0}}\right)^{2}\left\{\left(a e^{i \omega_{0} t_{0}}\left(e^{-i \omega_{0} t}-e^{-i \omega_{0} t^{\prime}}\right)+a^{\dagger} e^{-i \omega_{0} t_{0}}\left(e^{i \omega_{0} t}-e^{i \omega_{0} t^{\prime}}\right)\right)^{2}\right. \\
& \left.\quad-\left[2 i \omega_{0}\left(t-t^{\prime}-N \tau_{0}\right)+e^{i \omega_{0}\left(t-t^{\prime}\right)}-e^{-i \omega_{0}\left(t-t^{\prime}\right)}\right]\right\} \equiv 1+\mathcal{S}^{(1)}\left(t, t^{\prime}\right)+\mathcal{S}^{(2)}\left(t, t^{\prime}\right) .
\end{aligned}
$$

Without an explicit knowledge of $g^{\prime}\left(t_{1}\right)$ one will not be able to complete the integral appearing in the exponent.

\section{APPENDIX B: CORRECTIONS FOR LESSER GREEN'S FUNCTIONS IN TIME-DEPENDENT LANG-FIRSOV MODEL}

The first- and second-order corrections for lesser Green's function given in Eq. (3.7) are explicitly shown in this Appendix. At first order of $\mathcal{S}$, the lesser Green's function reads

$$
\begin{aligned}
G^{<,(1)}\left(t, t^{\prime}\right)= & -i e^{-\int_{t^{\prime}}^{t} d t_{1}\left(\bar{\varepsilon}\left(t_{1}\right)-\frac{\left|g^{\prime}\left(t_{1}\right)\right|^{2}}{\omega_{0}}\right)}\left[\left\langle\alpha\left|\mathcal{S}^{(1)}\left(t_{0}, t^{\prime}\right) X^{\dagger}\left(t^{\prime}\right) X(t)\right| \alpha\right\rangle+\left\langle\alpha\left|X^{\dagger}\left(t^{\prime}\right) X(t) \mathcal{S}^{(1)}\left(t, t_{0}\right)\right| \alpha\right\rangle\right] \\
= & \frac{i}{\omega_{0}} G^{<,(0)}\left(t, t^{\prime}\right)\left\{g^{\prime}\left(t_{0}\right)\left(e^{i \omega_{0} t^{\prime}}-e^{i \omega_{0} t_{0}}\right) e^{-i \omega_{0} t_{0}} \alpha^{*}+g^{\prime}\left(t_{0}\right)\left(e^{-i \omega_{0} t^{\prime}}-e^{-i \omega_{0} t_{0}}\right) e^{i \omega_{0} t_{0}}\left[\alpha+g\left(t^{\prime}\right) e^{i \omega_{0} t^{\prime}}-g(t) e^{i \omega_{0} t}\right]\right. \\
& \left.-g^{\prime}(t)\left(e^{-i \omega_{0} t}-e^{-i \omega_{0} t_{0}}\right) e^{i \omega_{0} t_{0}} \alpha-g^{\prime}(t)\left(e^{i \omega_{0} t}-e^{i \omega_{0} t_{0}}\right) e^{-i \omega_{0} t_{0}}\left[\alpha^{*}+g(t) e^{-i \omega_{0} t}-g\left(t^{\prime}\right) e^{-i \omega_{0} t^{\prime}}\right]\right\} .
\end{aligned}
$$

At second order of $\mathcal{S}$,

$$
\begin{aligned}
G^{<,(2)}\left(t, t^{\prime}\right) & =-i e^{-i \int_{t^{\prime}}^{t} d t_{1} \bar{\varepsilon}\left(t_{1}\right)}\left\{\left\langle\alpha\left|\mathcal{S}^{(2)}\left(t_{0}, t^{\prime}\right) X^{\dagger}\left(t^{\prime}\right) X(t)\right| \alpha\right\rangle+\left\langle\alpha\left|X^{\dagger}\left(t^{\prime}\right) X(t) \mathcal{S}^{(2)}\left(t, t_{0}\right)\right| \alpha\right\rangle+\left\langle\alpha\left|\mathcal{S}^{(1)}\left(t_{0}, t^{\prime}\right) X^{\dagger}\left(t^{\prime}\right) X(t) \mathcal{S}^{(1)}\left(t, t_{0}\right)\right| \alpha\right\rangle\right\} \\
& =G^{<,(2,1)}\left(t, t^{\prime}\right)+G^{<,(2,2)}\left(t, t^{\prime}\right)+G^{<,(2,3)}\left(t, t^{\prime}\right),
\end{aligned}
$$

where

$$
\begin{aligned}
G^{<,(2,1)}\left(t, t^{\prime}\right)= & -\frac{1}{2 \omega_{0}^{2}} G^{<,(0)}\left(t, t^{\prime}\right)\left\{\left(g^{\prime}\left(t_{0}\right)\right)^{2}\left(e^{i \omega_{0} t_{0}}-e^{i \omega_{0} t^{\prime}}\right)^{2} e^{-2 i \omega_{0} t_{0}}\left(\alpha^{*}\right)^{2}\right. \\
& +\left[g^{\prime}\left(t_{0}\right)\right]^{2}\left(e^{-i \omega_{0} t_{0}}-e^{-i \omega_{0} t^{\prime}}\right)^{2} e^{2 i \omega_{0} t_{0}}\left(\alpha+g\left(t^{\prime}\right) e^{i \omega_{0} t^{\prime}}-g(t) e^{i \omega_{0} t}\right)^{2} \\
& +\left[g^{\prime}\left(t_{0}\right)\right]^{2}\left(e^{-i \omega_{0} t_{0}}-e^{-i \omega_{0} t^{\prime}}\right)\left(e^{i \omega_{0} t_{0}}-e^{i \omega_{0} t^{\prime}}\right)\left[1+2 \alpha^{*}\left(\alpha+g\left(t^{\prime}\right) e^{i \omega_{0} t^{\prime}}-g(t) e^{i \omega_{0} t}\right)\right] \\
& \left.-\left[g^{\prime}\left(t_{0}\right)\right]^{2}\left[2 i \omega_{0}\left(t_{0}-t^{\prime}-N^{\left(t_{0}, t^{\prime}\right)} \tau\right)+e^{i \omega_{0}\left(t_{0}-t^{\prime}\right)}-e^{-i \omega_{0}\left(t_{0}-t^{\prime}\right)}\right]\right\}, \\
G^{<,(2,2)}\left(t, t^{\prime}\right)=- & \frac{1}{\omega_{0}^{2}} G^{<,(0)}\left(t, t^{\prime}\right)\left\{g^{\prime}\left(t_{0}\right) g^{\prime}(t)\left(e^{i \omega_{0} t_{0}}-e^{i \omega_{0} t^{\prime}}\right)\left(e^{-i \omega_{0} t}-e^{-i \omega_{0} t_{0}}\right)|\alpha|^{2}\right. \\
+ & g^{\prime}\left(t_{0}\right) g^{\prime}(t)\left(e^{i \omega_{0} t_{0}}-e^{i \omega_{0} t^{\prime}}\right)\left(e^{i \omega_{0} t}-e^{i \omega_{0} t_{0}}\right) e^{-2 i \omega_{0} t_{0}} \alpha^{*}\left(\alpha^{*}+g(t) e^{-i \omega_{0} t}-g\left(t^{\prime}\right) e^{-i \omega_{0} t^{\prime}}\right) \\
+ & g^{\prime}\left(t_{0}\right) g^{\prime}(t)\left(e^{-i \omega_{0} t_{0}}-e^{-i \omega_{0} t^{\prime}}\right)\left(e^{-i \omega_{0} t}-e^{-i \omega_{0} t_{0}}\right) e^{2 i \omega_{0} t_{0}} \alpha\left(\alpha+g\left(t^{\prime}\right) e^{i \omega_{0} t^{\prime}}-g(t) e^{i \omega_{0} t}\right) \\
+ & g^{\prime}\left(t_{0}\right) g^{\prime}(t)\left(e^{-i \omega_{0} t_{0}}-e^{-i \omega_{0} t^{\prime}}\right)\left(e^{i \omega_{0} t}-e^{i \omega_{0} t_{0}}\right)\left[\left(\alpha^{*}+g(t) e^{-i \omega_{0} t}-g\left(t^{\prime}\right) e^{-i \omega_{0} t^{\prime}}\right)\right. \\
& \left.\left.\times\left(\alpha+g\left(t^{\prime}\right) e^{i \omega_{0} t^{\prime}}-g(t) e^{i \omega_{0} t}\right)+1\right]\right\},
\end{aligned}
$$




$$
\begin{aligned}
G^{<,(2,3)}\left(t, t^{\prime}\right)= & -\frac{1}{\omega_{0}^{2}} G^{<,(0)}\left(t, t^{\prime}\right)\left\{\left[g^{\prime}(t)\right]^{2}\left(e^{-i \omega_{0} t}-e^{-i \omega_{0} t_{0}}\right)^{2} e^{2 i \omega_{0} t_{0}} \alpha^{2}\right. \\
& +\left[g^{\prime}(t)\right]^{2}\left(e^{i \omega_{0} t}-e^{i \omega_{0} t_{0}}\right)^{2} e^{-2 i \omega_{0} t_{0}}\left(\alpha^{*}+g(t) e^{-i \omega_{0} t}-g\left(t^{\prime}\right) e^{-i \omega_{0} t^{\prime}}\right)^{2} \\
& +\left[g^{\prime}(t)\right]^{2}\left(e^{-i \omega_{0} t}-e^{-i \omega_{0} t_{0}}\right)\left(e^{i \omega_{0} t}-e^{i \omega_{0} t_{0}}\right)\left[1+2 \alpha\left(\alpha^{*}+g(t) e^{-i \omega_{0} t}-g\left(t^{\prime}\right) e^{-i \omega_{0} t^{\prime}}\right)\right] \\
& \left.-\left[g^{\prime}(t)\right]^{2}\left[2 i \omega_{0}\left(t-t_{0}-N^{\left(t, t_{0}\right)} \tau\right)+e^{i \omega_{0}\left(t-t_{0}\right)}-e^{-i \omega_{0}\left(t-t_{0}\right)}\right]\right\} .
\end{aligned}
$$

Here, $N^{\left(t, t^{\prime}\right)}$ is defined as the quotient of dividing $t-t^{\prime}$ with $\tau=2 \pi / \omega_{0}$.

[1] L. D. Landau, Phys. Z. Sowjetunion 2, 46 (1932).

[2] E. Majorana, Nuovo Cimento 9, 43 (1932).

[3] C. Zener, Proc. R. Soc. A 137, 696 (1932).

[4] J. H. Shirley, Phys. Rev. 138, B979 (1965).

[5] H. Sambe, Phys. Rev. A 7, 2203 (1973).

[6] A.-P. Jauho, N. S. Wingreen, and Y. Meir, Phys. Rev. B 50, 5528 (1994).

[7] S. Kohler, T. Dittrich, and P. Hänggi, Phys. Rev. E 55, 300 (1997).

[8] M. Grifoni and P. Hänggi, Phys. Rep. 304, 229 (1998).

[9] M. Galperin, A. Nitzan, and M. A. Ratner, Phys. Rev. B 74, 075326 (2006).

[10] M. Galperin, M. A. Ratner, and A. Nitzan, J. Phys.: Condens. Matter 19, 103201 (2007).

[11] R.-P. Riwar and T. L. Schmidt, Phys. Rev. B 80, 125109 (2009).

[12] R. C. Monreal, F. Flores, and A. Martin-Rodero, Phys. Rev. B 82, 235412 (2010).

[13] S. Maier, T. L. Schmidt, and A. Komnik, Phys. Rev. B 83, 085401 (2011).

[14] K. F. Albrecht, A. Martin-Rodero, R. C. Monreal, L. Mühlbacher, and A. Levy Yeyati, Phys. Rev. B 87, 085127 (2013).

[15] B. Dong, G. H. Ding, and X. L. Lei, Phys. Rev. B 88, 075414 (2013).

[16] E. Y. Wilner, H. Wang, M. Thoss, and E. Rabani, Phys. Rev. B 89, 205129 (2014).

[17] H. Dehghani, T. Oka, and A. Mitra, Phys. Rev. B 90, 195429 (2014).

[18] K. F. Albrecht, A. Martin-Rodero, J. Schachenmayer, and L. Mühlbacher, Phys. Rev. B 91, 064305 (2015).

[19] R. Seoane Souto, R. Avriller, R. C. Monreal, A. Martín-Rodero, and A. Levy Yeyati, Phys. Rev. B 92, 125435 (2015).

[20] M. V. Berry, Proc. R. Soc. A 392, 45 (1984).

[21] I. G. Lang and Y. A. Firsov, Zh. Eksp. Teor. Fiz. 43, 1843 (1962) [Sov. Phys. JETP 16, 1301 (1963)].
[22] C. K. Lee, J. Cao, and J. Gong, Phys. Rev. E 86, 021109 (2012).

[23] K. Le Hur, Understanding Quantum Phase Transitions, edited by Lincoln D. Carr (Taylor and Francis, Boca Raton, 2010), pp. 217-240.

[24] K. Le Hur, L. Henriet, A. Petrescu, K. Plekhanov, G. Roux, and M. Schiró, arXiv:1505.00167.

[25] R. van Leeuwen, N. E. Dahlen, G. Stefanucci, C.-O. Almbladh, and U. von Barth, Time-dependent Density Functional Theory (Springer, Berlin Heidelberg, 2006), pp. 33-59.

[26] A. P. Jauho, Introduction to the Keldysh Nonequilibrium Green Function Technique, Lecture Notes in Physics (Springer, New York, 2006).

[27] G. D. Mahan, Many-particle Physics (Springer Science \& Business Media, New York, 2013).

[28] J. K. Freericks, H. R. Krishnamurthy, and T. Pruschke, Phys. Rev. Lett. 102, 136401 (2009).

[29] Y. H. Wang, H. Steinberg, P. Jarillo-Herrero, and N. Gedik, Science 342, 453 (2013).

[30] F. Mahmood, C.-K. Chan, Z. Alpichshev, D. Gardner, Y. Lee, P. A. Lee, and N. Gedik, Nat. Phys. 12, 306 (2016).

[31] P. Werner and M. Eckstein, Phys. Rev. B 88, 165108 (2013).

[32] M. Sentef, A. F. Kemper, B. Moritz, J. K. Freericks, Z.-X. Shen, and T. P. Devereaux, Phys. Rev. X 3, 041033 (2013).

[33] T. Oka and H. Aoki, Phys. Rev. B 79, 081406 (2009).

[34] N. H. Lindner, G. Refael, and V. Galitski, Nat. Phys. 7, 490 (2011).

[35] B. M. Fregoso, Y. H. Wang, N. Gedik, and V. Galitski, Phys. Rev. B 88, 155129 (2013).

[36] L. D'Alessio and M. Rigol, Nat. Commun. 6, 8336 (2015).

[37] M. Neupane, S.-Y. Xu, Y. Ishida, S. Jia, B. M. Fregoso, C. Liu, I. Belopolski, G. Bian, N. Alidoust, T. Durakiewicz, V. Galitski, S. Shin, R. J. Cava, and M. Z. Hasan, Phys. Rev. Lett. 115, 116801 (2015).

[38] D. Fausti, R. I. Tobey, N. Dean, S. Kaiser, A. Dienst, M. C. Hoffmann, S. Pyon, T. Takayama, H. Takagi, and A. Cavalleri, Science 331, 189 (2011). 